\title{
PRINCIPALES FACTORES ORGANIZATIVOS QUE INFLUYEN EN LA SATISFACCIÓN ESTUDIANTIL DE LAS UNIVERSIDADES
}

\author{
MAIN ORGANIZATIONAL FACTORS THAT INFLUENCE STUDENT \\ SATISFACTION IN UNIVERSITIES
}

\author{
Andrés Palacio Fierro \\ Universidad Tecnológica Indoamérica \\ andrespalacio@uti.edu.ec \\ Patricio Arévalo Chávez \\ Universidad Tecnológica Indoamérica \\ patricioarevalo@uti.edu.ec \\ Jorge Guadalupe Lanas \\ Universidad Tecnológica Indoamérica \\ jorgeguadalupe@uti.edu.ec
}

RECIBIDO: 03 / 04 / 2017

APROBADO: 19 / 05 / 2017

\begin{abstract}
Resumen
La educación no es un servicio como el que brinda un hotel de 5 estrellas, porque un estudiante no siempre logra obtener lo que él o ella quiere (usualmente buenas calificaciones), debido a que no basta solamente con ir a clases o pagar una matrícula, adicionalmente estas personas deberán dar tiempo, dedicación y esfuerzo, aspectos por el que se diferencia del resto de bienes y servicios que se ofertan. Por esta razón, entre otras, hay opiniones opuestas sobre cómo los alumnos deben ser tratados en una universidad, sin embargo, ellos siguen siendo usuarios de un servicio, por tanto, deben ser satisfechos sus requerimientos. En consecuencia, el estudiante podrá ser tratado como "cliente, consumidor o coproductor, la verdad no importa como sea llamado, siempre y cuando alguien se preocupe por ellos" (Redding, 2005). Consecuentemente, el objetivo general de este estudio es el de realizar un análisis de los principales factores que influyan en la satisfacción de los estudiantes, con el propósito de contribuir al conocimiento que las universidades deberían tener sobre ellos. Para lo cual el alcance de la investigación se centra sólo en los factores más controlables por una universidad, a través de una revisión documental en la que se analizó una gran cantidad de aportes realizados sobre la satisfacción estudiantil por investigadores a nivel global, con el fin de alcanzar un estudio incluyente con carácter internacional.
\end{abstract}

\section{Palabras clave}

Satisfacción de los estudiantes, calidad en la enseñanza, personal de contacto universitario, servicio a los estudiantes, instalaciones universitarias.

\begin{abstract}
Education is not a 5-star hotel service because a student is not always able to get what he or she wants (usually good grades) because it is not enough just to go to class or pay a tuition, additionally, these people must give time, dedication and effort, aspects by which it differs from the rest of goods and services that are offered. For this reason, among others, there are opposing opinions about how students should be treated in a university, however, they are still users of a service, therefore, their requirements must be met. Consequently, the student can be
\end{abstract}


treated as "customer, consumer or co-producer, the truth no matter what it is called, as long as, someone cares for them" (Redding, 2005). Consequently, the general objective of this study is to perform an analysis of the main factors that influence student satisfaction, with the purpose of contributing to the knowledge that universities should have about them. For which, the scope of the research focuses only on the factors most controllable by a university, through a documentary review that analyzed a large amount of contributions made on student satisfaction by researchers at a global level, in order to achieve an inclusive study with an international view.

\section{Keywords}

Student satisfaction, teaching quality, university contact personnel, service to students, university facilities.

\section{Introducción}

Los estudiantes son el corazón de las universidades, y según Bini y Masserini (2015), una actividad muy importante para satisfacerles es cómo se organiza una institución de educación superior, o que tan bien gestiona los elementos de la administración, tales como: planificación, organización, dirección, coordinación y control; con el fin de poder brindar a los estudiantes una experiencia inolvidable, a través de materiales didácticos útiles, actividades relevantes, horarios convenientes, etc. Adicionalmente, Civitci (2015) añade que, para mejorar aún más esa experiencia, una universidad debe lograr que el estudiante se sienta parte integrante de la misma.

Consecuentemente, las universidades deberían asistir a cada estudiante como un miembro importante de aquellas, con el fin de integrarlo adecuadamente a la institución que pertenece. Además, éstas deberían propender a desarrollar un sentido de lealtad en los estudiantes, la misma, que en parte, es el resultado de su satisfacción que proviene de la calidad del servicio (Harris \& Goode, 2004). Sin embargo, no es tarea fácil, debido a que el valor percibido por los estudiantes sobre la calidad, la mayoría de veces cambia a medida que pasa el tiempo, así se encuentra que "los estudiantes que están en un nivel académico más alto buscan más educación de calidad y perfección del sistema" (Misanew \& Tadesse, 2014).

Adicionalmente, hay que tomar en cuenta que la educación es un servicio especial y único, porque tiene un alto contacto humano y una cambiante interacción bidireccional entre el profesor y el estudiante, que se traduce en una experiencia diferente en cada encuentro, la cual no se limita solamente al contacto con el docente, sino que incluye a todo el entorno donde estudia, por tal motivo, los factores escogidos para esta investigación fueron: la enseñanza, el personal de contacto con el servicio y las instalaciones de la universidad.

\section{Método}

Método Sistémico-complejo 


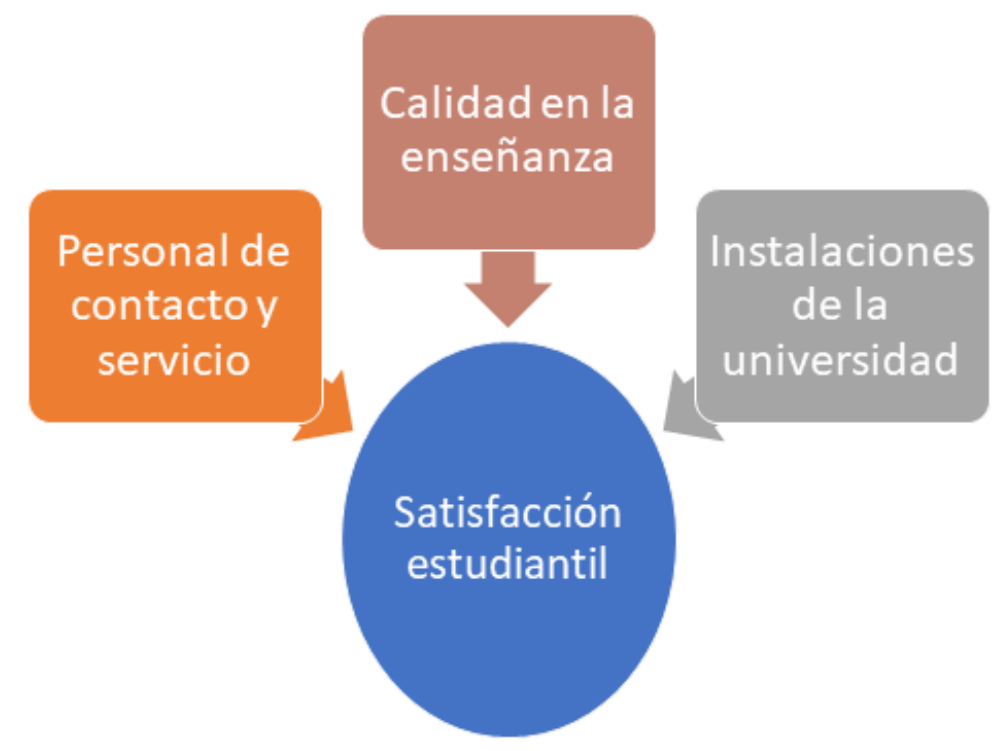

Figura 1: Principales factores organizativos que influyen en la satisfacción estudiantil de las universidades.

La metodología tiene como fuente a la información documental, a través de instrumentos de recolección bibliográfica, centrándose en artículos de revistas de categoría mundial entre Q4 a Q1, siendo el "análisis" el procedimiento para el tratamiento de la información investigada y cuya finalidad fue la aplicada.

\section{Resultados - Análisis}

Factor 1: calidad de la enseñanza.

La enseñanza es el primer factor descrito en esta investigación, debido a que es una actividad muy estudiada alrededor del mundo, ver, por ejemplo, a Bini y Masserini (2015). Inclusive, podría ser considerado como el factor más influyente en la satisfacción de los estudiantes (Butt \& Rehman, 2010). Esta relación entre la calidad de la enseñanza y la satisfacción de los estudiantes también es confirmada por Rapert, Smith, Velliquette y Garretson (2004), quienes afirman que "la calidad de la enseñanza es alta cuando los estudiantes están satisfechos".

Pero; ¿qué es "calidad"? Chonko, Tanner y Davis (2002) indican que la calidad es inherente a la distinción de una universidad, cuando alcanza cierto nivel, medida por alguna característica de servicio y, a la vez, esté de acuerdo con las expectativas de los estudiantes. Además, la calidad se realiza cuando la satisfacción del cliente se maximiza, es decir, en la ocasión que un producto o servicio se ajusta a su uso previsto (Smimou \& Dahl, 2012). A pesar de estas definiciones, persisten diferentes puntos de vista sobre la calidad, especialmente cuando se compara la educación superior y la industria, donde la última tiene un producto final muy definido (Tang \& Hussin, 2011).

Cabe mencionar que, para conocer el nivel de la calidad en la enseñanza, la herramienta de medición más habitual para evaluar el rendimiento de un instructor es a través de la información proporcionada por los mismos estudiantes. Sin embargo, "dada la posibilidad de que los resultados derivados de la información reportada por los estudiantes no sean completamente objetivos, es necesario combinar estas fuentes con otras" (Rosendo-Ríos, Messia-de-la-Cerda, \& Laguna-Sánchez, 2013).

Las encuestas y las entrevistas a los estudiantes como fuente de información son ampliamente utilizadas, y a través de ellas, Rivkin, Hanushek y Kain (2005) revelaron que la calidad de la enseñanza y un mayor rendimiento estudiantil son más observables en los profesores con experiencia. A pesar del hallazgo anterior, Maulana, Helms-Lorenz y Van de Grift (2015) demostraron que no todos los comportamientos de los profesores sin experiencia tenían un 
desempeño inferior al de los experimentados. Por ejemplo, estos autores mencionaron que los profesores sin experiencia son mejores en comportamientos de enseñanza, que representan un grado de dificultad menor (es decir, en los climas de aprendizaje y las instrucciones claras), pero, todavía tienen un nivel inferior con respecto a los docentes experimentados en relación a las competencias más complejas (es decir, adaptación y estrategias de enseñanza).

En lo referente al manejo de aula, Opdenakker and Minnaert (2011) mencionaron que esta actividad tiene que ser efectiva y eficiente con el objetivo de asegurar los siguientes puntos: el horario de las clases; el syllabus, el cual es parte de la guía de enseñanza; el performance de los profesores; la maximización de las tareas principales y la minimización de otras tareas; la estructuración y preparación de la clase día a día; la administración de la clase misma; y muchas otras.

En lo referente a la guía de enseñanza, Rosendo-Ríos, Messia-de-la-Cerda y Laguna-Sánchez (2013) amplificaron los elementos básicos que la conforman, incluyendo a las actividades de capacitación como seminarios o talleres, los mismos que podrían ser elaborados por el director o decano de la facultad o por los mismos docentes de cada carrera. Estos autores también aclaran que, el cronograma para las actividades propuestas en la guía es muchas veces sólo una aproximación, debido a los cambios de las condiciones cuando éste es implementado.

Cabe añadir, que la administración del curso no es una tarea fácil para el profesor, en particular porque ellos tienen que enfrentar un sin número expectativas y comportamientos impredecibles de los estudiantes. Por lo que, una efectiva comunicación debe ser un hecho, principalmente cuando está relacionada con lo quiere el docente en la clase, algo similar a la de un controlador de un aeropuerto, quien debe transmitir instrucciones muy claras (Mundschenk, Miner, \& Nastally, 2011).

Para mejorar esta comunicación, existen herramientas educacionales, así como métodos y estrategias de enseñanza. De acuerdo a Roman (2014), el sistema tradicional es usualmente utilizado por muchos profesores, pero el mismo no dispone con la retroalimentación de los estudiantes, por esta razón, el marco de referencia recomendado debe incluir la participación de los estudiantes en las actividades de aprendizaje y en los métodos de enseñanza, para eso se necesita una gran dosis de creatividad, como puede ser el uso de ejemplos relevantes y la tecnología, con el fin de lograr cautivarles. En este sentido, este involucramiento también puede obtenerse mediante la discusión, utilizando pequeños grupos para la resolución de problemas, creando preguntas, etc.

Complementado, la motivación puede ser usada por los profesores como una estrategia, la cual es requerida por los estudiantes, como resultado, ellos ponen más atención y alcanzan mejoras en los procesos de información recibida (Pugh \& Bergin, 2006). Cuando ellos se encuentran motivados pueden concentrar sus esfuerzos para entender el material de la clase -para aprender con un significado- o cómo podrían utilizarlo en sus vidas (Roman, 2014).

Consecuentemente, el aprendizaje con aplicaciones reales es esencial para la satisfacción general de los estudiantes, aunque la responsabilidad de este proceso no es exclusiva de los docentes, también es de los estudiantes, de las autoridades de la universidad, y de todos aquellos que de alguna forma están involucrados en el mismo (Rosendo-Ríos, Messia-de-la-Cerda, \& Laguna-Sánchez, 2013).

Factor 2: Personal de contacto y servicio.

Los estudiantes a tiempo completo tienen una gran cantidad de interacción con el personal de contacto de su institución educativa, por tal razón algunos investigadores han demostrado que este personal y su servicio también son un factor importante a ser valorado. 
Pero; ¿Qué es servicio? Se define como la ejecución de los procesos, incluyendo al conjunto de actividades interrelaciones para crear valor al usuario (Banwet \& Datta, 2002). Consecuentemente, si una institución educativa quiere ofrecer un servicio de alta calidad, ésta deberá administrar los procesos efectivamente, así podrá evidenciar que sus resultados se relacionan con la satisfacción de las necesidades de sus estudiantes.

Para alcanzar este propósito, entre otros, un método probado para asegurar la calidad de los procesos en una universidad y su valor para los estudiantes es el SERVQUAL, el cual es ampliamente mencionado como una herramienta de medición que analiza la calidad del servicio en las instituciones de educación. (ver, por ejemplo, Rodrigues, Barkur, Varambally \& Motlagh, 2011; Arslan \& Akkas, 2014; Sadeh \& Garkaz, 2015).

Como resultado, se alcanzará uno de los objetivos que es el de proveer un servicio de excelencia, además, al mismo tiempo, se intentará resolver cualquier problema que tenga un estudiante inmediatamente (Zeithaml, Bitner, \& D Gremler, 2009). Entonces, las habilidades interpersonales y la reacción que se tenga ante los problemas de los alumnos, representan lo esencial para brindar un servicio de calidad en lo que se refiere a la satisfacción percibida por el alumno (El-Hilali \& Al-Jaber, 2015).

A pesar de que el personal de contacto tiene una gran responsabilidad para alcanzar este objetivo, hay que añadir que ellos no trabajan solos. Por tal motivo, esta responsabilidad debería incluir a todo el personal y la organización en sí, como un todo integral del servicio brindado a los estudiantes.

Adicionalmente, los administradores de las instituciones deberían también estar involucrados en poner especial atención con las percepciones de los estudiantes, en lo relacionado a la satisfacción de la calidad del servicio (Lenton, 2015). Además, complementa este autor, que esa satisfacción está correlacionada con el ambiente de aprendizaje, el cual no nace por sí solo, ya que necesita de acciones encaminadas a esa meta, como el de propender la satisfacción del personal que trabaja en la organización, para que, desde ahí, el ambiente sea agradable y positivo (Gounaris, 2006).

Para alcanzar este fin, las universidades deberían pensar en esos medios que permiten satisfacer las necesidades de sus clientes internos y así lograr el ambiente indicado anteriormente, conjuntamente, si añade una capacitación al personal para que toda la organización tenga claro el objetivo de mejorar el nivel satisfacción de sus estudiantes, entre otras medidas dirigidas a los empleados, se alcanzará la meta propuesta, empujada por ese mayor compromiso de todos quienes conforman la institución. (Tortosa Edo, Llorens-Monzonís, Moliner-Tena, \& SánchezGarcía, 2010).

Asimismo, varios investigadores también vieron la necesidad de que la administración de una organización deba ser gestionada en un modo efectivo y eficiente; además, que disponga de un sistema de reclutamiento del personal eficaz y facilite su capacitación continua, para que los empleados continúen motivados y comprometidos. (Khan \& Matlay, 2009)

Por último, el servicio que se brinda a los estudiantes es importante en cada uno de los encuentros, ya sea en el salón de clase, en la biblioteca, en la cafetería, en el centro médico, en el centro deportivo, etc. (Arslan \& Akkas, 2014).

Factor 3: Instalaciones de la Universidad.

Las instalaciones universitarias apoyan al servicio que brinda el personal y a la vez afecta directamente a todas las interacciones que se ejecutan en ella. Pero; ¿Qué tan importante es este factor? En el estudio de Arslan y Akkas (2014) sobre las instalaciones, se llegó a la conclusión de que la importancia de este factor sólo tenía un nivel medio, relativo a la satisfacción general de los alumnos, aunque en la investigación empírica de Negricea, Edu y Avram (2014), 
concluyeron que este factor si tenía un gran impacto en la satisfacción de los alumnos, sobre todo cuando estas instalaciones se materializan como elementos tangibles.

Estos elementos y el entorno físico del campus son la primera impresión del estudiante, y pueden causar un gran impacto en la satisfacción del alumnado. Sin embargo, esto no siempre es cierto, ya que las primeras impresiones de los estudiantes podrían provenir de la reputación de la universidad, la misma que proviene la mayor parte del tiempo a través de la comunicación boca-oreja. Sin embargo, la primera visualización del entorno del campus y sus características si influyen en la percepción del estudiante potencial (Lenton, 2015).

A continuación, se analizan con más detalle los elementos tangibles más relacionados con una universidad:

Salón de clases

Mientras mayor comodidad en las aulas (incluyendo sillas adecuadas, iluminación, buen equipo y materiales necesarios), mayor número de estudiantes satisfechos (El-Hilali \& Al-Jaber, 2015). Sobre este tema ya existen algunos acuerdos sobre los requisitos mínimos que un aula debería tener, como: evitar temperaturas extremas, alcanzar una buena visibilidad, acústica, calidad del aire, iluminación y un mantenimiento adecuado (Yang, Becerik-Gerber, \& Mino, 2013). En la actualidad, la inclusión de algunas herramientas tecnológicas, como disponer de computador, proyector e internet, son básicas para el aula de clase. (Yang, Becerik-Gerber, \& Mino, 2013).

\section{Tecnología}

Como se mencionó anteriormente, la tecnología está presente en las aulas, pero, de hecho, está involucrada en todas las instalaciones del campus, desde una comunicación personalizada interpersonal que conecta a los estudiantes con los profesores y personal administrativo, hasta nuevos métodos para mejorar las experiencias de aprendizaje y los procesos administrativos, como el autoservicio en la matrícula, atención al cliente, calificaciones, certificados, entre otros. De hecho, este factor es percibido por los estudiantes como una de las herramientas de más vanguardia que dispone una universidad (Arif \& Ilyas, 2011).

\section{Biblioteca}

Hoy en día, hay bibliotecas en línea (online) y bibliotecas físicas; por un lado, en la física, una queja común es sobre las copias insuficientes para todos los estudiantes, otra queja, que ha causado su insatisfacción, es sobre el ruido, especialmente en los espacios de estudio; por otro lado, en la online, los estudiantes sollozan cuando no es fácil navegar a través de la biblioteca virtual o cuando es muy difícil encontrar buenos libros electrónicos y artículos. Sin embargo, se complacen cuando tienen una visita guiada que explica todos los detalles de ambos tipos de bibliotecas, incluyendo el uso de impresoras y copiadoras (Fennell, Stinson, \& Okolo, 2012)

\section{Conclusiones}

Según la literatura revisada, la satisfacción de los alumnos difiere en múltiples aspectos, desde el nivel en el que cada estudiante está estudiando, la ubicación de la institución de educación superior, aún más, con las innumerables expectativas de cada estudiante.

Sin embargo, en forma general, las universidades pueden influir directamente en la satisfacción de su población estudiantil, a través de las acciones propositivas en al menos de los principales factores que generan satisfacción a los estudiantes, los cuales son:

En primer lugar, la calidad de la enseñanza, que se refleja en los resultados de la relación profesor-alumno (Guolla, 1999). Además, es considerado como "el factor más influyente entre todos" (Butt \& Rehman, 2010).

En segundo lugar, excelentes servicios, junto con la oportunidad de resolver cualquier inquietud de inmediato, de manera personalizada y en un entorno motivador; los mismos que son 
brindados por el personal de contacto, pero con el apoyo de todos los empleados y la organización en forma integral.

En tercer lugar, las instalaciones de la universidad, al respecto Negricea, Edu y Avram (2014) afirmaron que este factor tiene un gran impacto en la satisfacción, especialmente cuando estas instalaciones se materializan en elementos útiles y tangibles para los estudiantes, como un salón de clases con sillas cómodas, buena iluminación, temperatura adecuada y visibilidad; además de la implementación de tecnología tanto dentro como fuera del aula, la cual pueda mejorar las experiencias de aprendizaje y los procesos administrativos; sin olvidar la optimización donde los estudiantes tienen interacción, como las bibliotecas, cafetería, centro deportivo, dispensario médico, etc., a través del conocimiento de los requerimientos de sus alumnos.

Por último, cuando un servicio integral es mal proporcionado, los estudiantes pueden percibir si una institución está bien organizada o no para brindar este servicio de una manera apropiada. Al respecto Bini y Masserini (2015) afirmaron, la administración y la gestión efectiva y eficiente de todos los factores mencionados, incluyendo su interrelación, tienen un papel muy importante para satisfacer a los estudiantes, a fin de ofrecerles una experiencia perdurable; si las instituciones de educación lo logran, alcanzarían el objetivo de mejorar la satisfacción estudiantil.

\section{Referencias bibliográficas}

Arif, S., \& Ilyas, M. (2011). Leadership, empowerment and customer satisfaction in teaching institutions; Case study of a Pakistani University. The TQM Journal, 23(4), 388-402. doi:10.1108/17542731111139473

Arslan, S., \& Akkas, O. (2014). Quality of College Life (QCL) of Students in Turkey: Students' Life Satisfaction and Identification. Social Indicators Research, 115(2), 869-864. doi:10.1007/s11205-013-0235-9

Banwet, D., \& Datta, B. (2002). Effect of service quality on post-visit intentions over time: The case of a library. Total Quality Management, 13(14), 537-546. doi:10.1080/09544120220149322

Bini, M., \& Masserini, L. (2015). Student's Satisfaction and Teaching Efficiency of University Offer. Social Indicators Research, 1-16. doi:10.1007/s11205-015-1141-0

Butt, B. Z., \& Rehman, K. U. (2010). A study examining the students satisfaction in higher education. Procedia - Social and Behavioral Sciences, 2(2), 5446-5450. doi:10.1016/2010.03.888

Chonko, L. B., Tanner, J. F., \& Davis, R. (2002). What are they thinking? Students' expectations and self-assessments. Journal of Education for Business, 77(5), 271-281. doi:10.1080/08832320209599676

Cornelius-White, J. (2007 de 2007). Learner-centered teacher-student relationships are effective: a meta-analysis. Review of Educational Research, 77(1), 113-143. doi: $10.3102 / 003465430298563$

Douglas, J., Douglas, A., \& Barnes, B. (2006). Measuring student satisfaction at a UK university. Quality Assurance in Education, 14(3), 251-267. doi:10.1108/09684880610678568

El-Hilali, N., \& Al-Jaber, S. H. (2015). Procedia - Social and Behavioral Sciences . Students' Satisfaction and Achievement and Absorption Capacity in Higher Education, 177(1), 420-427. doi: 10.1016/j.sbspro.2015.02.384

El-Said, O. A., \& Fathy, E. A. (2015). Assessing university students' satisfaction with oncampus cafeteria services. Tourism Management Perspectives, 26(1), 318-324. doi:10.1016/j.tmp.2015.09.006

Fennell, G., Stinson, R., \& Okolo, S. N. (2012). Getting to the root of student 'dis-satisfaction' at the University of East London. Focus, 56(1), 29-32. Obtenido de http://roar.uel.ac.uk/1770/1/12_7.pdf 
Gounaris, S. P. (2006). Internal-market orientation and its measurement. Journal of Business Research, 59(4), 432-448. doi:10.1016/j.jbusres.2005.10.003

Guolla, M. (1999). Assessing the Teaching Quality to Student Satisfaction Relationship: Applied Customer Satisfaction Research in the Classroom. Journal of Marketing Theory and Practice, 7(3), 87-97. doi:10.1080/10696679.1999.11501843

Harris, L. C., \& Goode, M. M. (2004). The four levels of loyalty and the pivotal role of trust: a study of online service dynamics. Journal of Retailing, 80(2), 139-158. doi:doi:10.1016/j.jretai.2004.04.002

Hendrickson, B., Rosen, D., \& Aune, R. K. (2011). An analysis of friendship networks, social connectedness, homesickness, and satisfaction levels of international students. International Journal of Intercultural Relations, 35(3), 281-295. doi:10.1016/j.ijintrel.2010.08.001

Khan, H., \& Matlay, H. (2009). Implementing service excellence in higher education. Education + Training, 51((8-9)), 769-780. doi:10.1108/00400910911005299

Lenton, P. (2015). Determining student satisfaction: An economic analysis of the National Student Survey. Economics of Education Review, 47(1), 118-127. doi:10.1016/j.econedurev.2015.05.001

Maulana, R., Helms-Lorenz, M., \& Van de Grift, W. (2015). A longitudinal study of induction on the acceleration of growth in teaching quality of beginning teachers through the eyes of their students. Teaching and Teacher Education, 51, 225-245. doi:10.1016/j.tate.2015.07.003

Misanew, A., \& Tadesse, M. (2014). Determinants of Student and Staff Satisfaction with Services at Dilla University, Ethiopia: Application of Single and Multilevel Logistic Regression Analyses. Social Indicators Research, 119(3), 1571-1587. doi:10.1007/s11205-013-0561-y

Mundschenk, N. A., Miner, C. A., \& Nastally, B. L. (2011). Effective Classroom Management. An Air Traffic Control Analogy. Intervention in School and Clinic, 47(2), 98-103. doi: $10.1177 / 1053451211414190$

Negricea, C. I., Edu, T., \& Avram, E. M. (2014). Establishing Influence of Specific Academic Quality on Student Satisfaction. Procedia - Social and Behavioral Sciences , 116(1), 4430 - 4435 . doi:10.1016/j.sbspro.2014.01.961

Opdenakker, M., \& Minnaert, A. (2011). Relationship between learning environment characteristics and academic engagement 1. Psychological Reports, 109(1), 259-284. doi:10.2466/09.10.11.PR0.109.4.259-284

Pugh, K. J., \& Bergin, D. A. (2006). Motivational Influences on Transfer. Educational Psychologist, 41(3), 147-160. doi:10.1207/s15326985ep4103_2

Rapert, M., Smith, S., Velliquette, A., \& Garretson, J. (2004). The Meaning of Quality: Expectations of Students in Pursuit of an MBA . Journal of Education for Business, 80(1), 17-24. doi:10.3200/JOEB.80.1.17-24

Reavill, L. R. (1997). Quality assessment and the stakeholder model of higher education. 246252, 8((2-3)). doi:10.1080/09544129710143

Rodrigues, L., Barkur, G., Varambally, K., \& Motlagh, F. (2011). Comparison of SERVQUAL and SERVPERF metrics: an empirical study. The TQM Journal, 629-643. doi:10.1108/17542731111175248

Roman, I. (2014). Qualitative Methods for Determining Students' Satisfaction with Teaching Quality . Procedia - Social and Behavioral Sciences, 149(1), 825 - 830 . doi:10.1016/j.sbspro.2014.08.320

Rosendo-Ríos, V., Messia-de-la-Cerda, J., \& Laguna-Sánchez, P. (2013). Proposal of a model of teaching quality assessment at university level. Procedia - Social and Behavioral Sciences, 83(1), 883 - 894 . doi:10.1016/j.sbspro.2013.06.166

Sadeh, E., \& Garkaz, M. (2015). Explaining the mediating role of service quality between quality management enablers and students' satisfaction in higher education institutes: the perception of managers. Total Quality Management \& Business Excellence, 26((1112)), 1335-1356. doi:10.1080/14783363.2014.931065 
Smimou, K., \& Dahl, D. W. (2012). On the Relationship Between Students' Perceptions of Teaching Quality, Methods of Assessment, and Satisfaction. Journal of Education for Business, 87(1), 22-35. doi: 10.1080/08832323.2010.550339

Tang, S., \& Hussin, S. (2011). Quality in Higher Education: A Variety of Stakeholder Perspectives. International Journal of Social Science and Humanity, 1(2), n/a. doi:10.7763/IJSSH.2011.V1.21

Tortosa Edo, V., Llorens-Monzonís, J., Moliner-Tena, M. Á., \& Sánchez-García, J. (2010). The influence of internal market orientation on external outcomes: The mediating role of employees' attitudes. The services industries journal, 30(8), 1279-1297. doi:http://dx.doi.org/10.1108/JSTP-11-2013-0259

Yang, Z., Becerik-Gerber, B., \& Mino, L. (2013). A study on student perceptions of higher education classrooms: Impact of classroom attributes on student satisfaction and performance. Building and Environment, 70(1), 171-188. doi:10.1016/j.buildenv.2013.08.030

Zeithaml, B., Bitner, M., \& D Gremler, D. (2009). Services marketing : integrating customer focus across the firm. (3rd. ed.). London: McGraw-Hill/Irwin . 